\title{
La red de facilitadores de los "Espacios para Crecer” en Barranquilla (Colombia). Estrategias de continuidad, ajuste comunitario y mejora de la implementación en los programas de prevención del trabajo infantil.
}

Isidro Maya-Jariego ${ }^{1}$

Jorge E. Palacio²

Reporte de una intervención psico-educativa

Material original autorizado para su primera publicación en el Journal de Ciencias Sociales, Revista Académica de la Facultad de Ciencias Sociales de la Universidad de Palermo

Recibido: 25-2-2014

Aceptado: 3-5-2014

\section{Resumen}

Los "Espacios para Crecer" y el "Baúl de Juegos" son dos prácticas educativas basadas en la evidencia que se han mostrado efectivas en la prevención del trabajo infantil. La iniciativa "Edúcame Primero, Colombia" consistió en aplicar las actividades lúdicas, educativas y de desarrollo psicológico con menores trabajadores a través de una red de facilitadores. La introducción de esta innovación, de carácter comunitario, mejoró la pertinencia de los materiales educativos y la implementación del programa. Los mediadores comunitarios facilitaron la implicación de las madres en las actividades preventivas, mejorando la conexión entre las familias y los centros educativos. También fortalecieron la implementación, involucrando al profesorado y mejorando el ajuste comunitario del programa a la institución educativa. A lo largo de la intervención, los facilitadores desarrollaron un mecanismo informal de trabajo en red, que facilitó el intercambio de buenas prácticas y la adaptación del programa a las peculiaridades de cada contexto comunitario. La experiencia muestra cómo las estrategias de ajuste comunitario en este caso no sólo mejoran la implementación y la efectividad del programa, sino que contribuyen a su sostenibilidad.

Palabras clave: trabajo infantil, prevención, buenas prácticas, mediadores comunitarios.

\footnotetext{
Abstract

Best practices and experiences of the facilitators of the program "Edúcame Primero Colombia" for prevention of Child Labor in Barranquilla. Sustainaibility, community fit and improvement of the implementation.

Edúcame Primero, Colombia is an evidence-based practice implemented in a network of schools and neighborhoods for preventing child labor. The intervention consists of academic orientation, socialization among peers and personal development activities, which are usually applied ${ }_{1}^{1}$ Doctor en Psicología. Profesor Titular del Departamento de Psicología Social de la Universidad de Sevilla (España). Investigador Principal del Laboratorio de Redes Personales y Comunidades (LRPC). Director de REDES. Revista Hispana para el Análisis de Redes Sociales. Esta experiencia participó en la convocatoria de buenas prácticas de la Red Latinoamericana Contra el Trabajo Infantil (LACTI) en su edición de 2013.

2 Doctor en Psicología. Profesor de la Universidad del Norte (Colombia). Coordinador del Programa de Doctorado en Psicología. Miembro del Grupo de Investigación en Desarrollo Humano. Coordinador del programa "Edúcame Primero, Colombia".
} 
in academic institutions following the instructions of the educational materials of the program. These set of activities are shown to be effective in previous applications of the program in 172 schools from Barranquilla, Cartagena and Santa Marta. The intervention is effective improving academic performance and reducing absenteeism at school. There is also evidence of an impact in the number of hours of child labor, as well as the number of children doing some labor activities. During the implementation of the program, a group of psychology graduate students was selected to coordinate the activities in each school participating in the intervention. Each student was assigned to a particular school and adopted the role of "facilitator", adjusting the program to the specifics of the context. The main task assigned to facilitators was to apply the educational materials of the program. However, during implementation they negotiated with the principal and teachers of the school, they were in contact with the families of children participating in the program, and they informally assumed a broker role between the school, the families and the neighborhood community organizations where the intervention was taking place. These activities were not originally planned in the program, but finally revealed to be essential for the successful implementation of the program. Facilitators assumed an unanticipated role of improving community adjustment of the intervention, adapting the program to the diversity of community contexts and schools.

Keywords: child labour, prevention, best practices, community mediators.

\section{Una red de agentes comunitarios para la prevención del trabajo infantil}

\section{Antecedentes de la intervención}

La experiencia que resumimos a continuación es una intervención psico-educativa para la reducción del trabajo infantil que se ha desarrollado durante los últimos 5 años en Colombia. Nos centramos en el caso específico de la aplicación del programa en Barranquilla, capital del departamento del Atlántico, donde la apropiación local del programa introdujo la figura de los facilitadores comunitarios. La actuación se lleva a cabo en barrios pobres, con una elevada prevalencia de menores trabajadores en negocios familiares, la venta de productos en la calle y la mendicidad. En muchos casos, el problema se relaciona con el abandono de la escuela y se ve afectado por las deficiencias de los servicios públicos y el sistema educativo. Las consecuencias de la violencia política, el desplazamiento interno de población y la reinserción de excombatientes se reflejan en las dinámicas familiares, presentando necesidades especiales para una parte de la población de menores trabajadores. El programa ha ido evolucionando en los últimos años, incorporando acciones de carácter comunitario junto con las actividades habituales en los centros educativos.

\section{Acciones: qué se hizo y cómo se hizo}

Edúcame Primero, Colombia se inicia como una práctica basada en la evidencia, implementando en una amplia red de centros educativos de diferentes regiones colombianas los "Espacios para Crecer" y el "Baúl de Juegos". La intervención consiste en un conjunto de 
actividades de orientación académica, socialización entre iguales y desarrollo personal, que normalmente se llevan a cabo en el colegio siguiendo fielmente las indicaciones de los materiales educativos del programa. Se trata de actividades de prevención del trabajo infantil que se han mostrado efectivas en experiencias de evaluación previas. El programa mejora el rendimiento académico y reduce el absentismo escolar. En la Costa Caribe se llevó a cabo en 172 centros educativos de Barranquilla, Cartagena y Santa Marta.

Edúcame Primero, Colombia se aplica a lo largo de tres años (2008-2010) como un proyecto de cooperación internacional en el que participan organizaciones colombianas y norteamericanas. Desde 2010 la Universidad del Norte (Barranquilla, Colombia) se hace cargo de la continuidad del programa, centrando los esfuerzos principalmente en el "Baúl de Juegos". Se trata de un proceso de apropiación local de la iniciativa de cooperación al desarrollo, que mantiene los componentes centrales de la intervención. El "Baúl de Juegos" funciona como una alternativa al trabajo infantil, facilitando un espacio seguro que compite con el tiempo dedicado a actividades laborales. Sin embargo, la versión local del programa también incorpora innovaciones. La Universidad del Norte establece una red de facilitadores que coordinan la aplicación del programa en cada centro. Los facilitadores promueven la aplicación del "Baúl de Juegos" en cada escuela, se coordinan con los directivos del centro, implican al profesorado, median con las familias y adaptan el programa a las peculiaridades de cada contexto comunitario.

En el 2011 se establece un nuevo marco de cooperación internacional entre el Centro de Investigación en Desarrollo Humano de la Universidad del Norte y el Laboratorio de Redes Personales y Comunidades de la Universidad de Sevilla. Desde entonces, estudiantes españolas participan cada año en estancias breves con Becas de Formación Solidaria de la Oficina de Cooperación al Desarrollo de la Universidad de Sevilla. Las cooperantes participan en la implementación del Baúl de Juegos, colaboran en la evaluación formativa del programa, desarrollan actividades del Practicum en Psicología y, en algunos casos, llevan a cabo las tareas de campo del Trabajo Fin de Máster (TFM) en Psicología de la Intervención Social y Comunitaria. Por su parte, el equipo internacional de evaluadores, en colaboración con los facilitadores comunitarios, puso en marcha actividades para mejorar la implementación del programa. También elaboró sendos estudios de caso sobre los "Espacios para Crecer" y el "Baúl de Juegos", siguiendo el modelo Getting to Outcomes. 


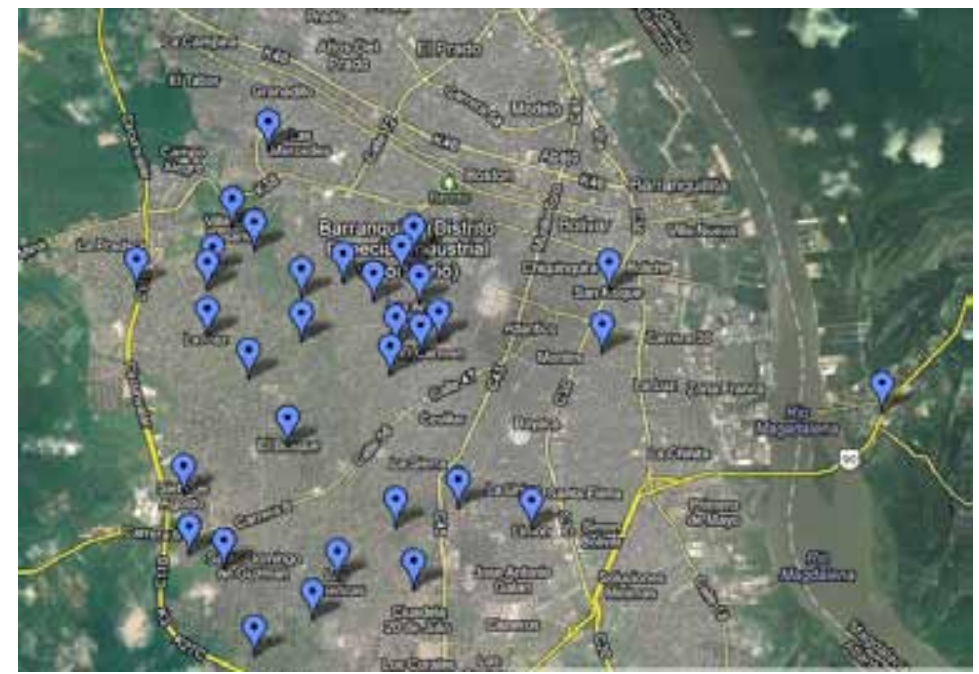

Figura 1. Centros escolares en los que se aplica Edúcame Primero Colombia en Barranquilla

Actualmente, el equipo internacional de evaluadores contribuye a la sostenibilidad del programa promoviendo innovaciones comunitarias. Concretamente, se están diseñando microintervenciones psico-educativas para mejorar la implementación del "Baúl de Juegos". También se está elaborando un catálogo de acciones comunitarias para la intervención en barrios, en las escuelas y en las familias. Se incorporan actividades para atender las necesidades especiales de las familias de excombatientes y de los desplazados por la violencia política en Colombia. Finalmente, se toman indicadores de preparación comunitaria para la transferencia del programa al área metropolitana de Lima (Perú), donde está previsto aplicar la intervención entre 2014 y 2016.

\section{Logros y buenas prácticas de intervención}

Esta experiencia se caracteriza por combinar (a) la evaluación formativa de un programa psico-educativo con (b) estrategias de ajuste comunitario y (c) actividades de sistematización de la práctica. La combinación de los tres tipos de acciones anteriores (d) mejoró la implementación del programa y (e) favoreció la continuidad en diferentes contextos de aplicación.

Las estrategias psico-educativas del programa Edúcame Primero, Colombia resultan efectivas en la reducción del trabajo infantil y en la mejora de la calidad de vida de los menores trabajadores. Cuando se aplican con suficiente intensidad, los "Espacios para Crecer" mejoran el rendimiento académico en lengua y matemáticas, aumentan las habilidades sociales, reducen el absentismo escolar y mejoran la autoestima de los participantes. Complementariamente, el "Baúl de Juegos" es un espacio alternativo que reduce el tiempo dedicado al trabajo infantil.

Sin embargo, es frecuente que los profesores de los centros en los que se aplica el programa perciban Edúcame Primero, Colombia como un elemento extraño, sobre el que no tienen influencia. Además, cuestionan el valor académico de las actividades lúdicas del "Baúl de Juegos". Los profesores preferirían dedicar más tiempo a la enseñanza de la lengua, las matemáticas y otras asignaturas regladas. Por su parte, es frecuente que las familias sean ajenas a las actividades de prevención del trabajo infantil que se realizan en la escuela.

Los facilitadores mejoraron el ajuste comunitario de la intervención, ejerciendo un rol de 
mediación con las escuelas y las familias. Cada facilitador se encargó de negociar con el director del colegio, adaptó las actividades y el funcionamiento del programa a las características del barrio, lidió con las objeciones que ponía el profesorado e intentó implicarlos en el desarrollo del programa. Otra actividad fundamental consistió en coordinarse con las familias, informando del contenido de la intervención, monitorizando la participación de los menores, involucrando a los padres en actividades específicas y promoviendo actitudes favorables hacia la implicación educativa y la prevención del trabajo infantil. Aunque en muchos casos suponía separarse de lo inicialmente planificado, las estrategias de ajuste de los facilitadores mejoraron en general la implementación del programa. Además, los facilitadores se coordinaron informalmente entre sí, aumentando la consistencia interna del programa.

La evaluación formativa del equipo internacional le ha dado continuidad a la experiencia de los facilitadores comunitarios. En primer lugar, ha documentado y sistematizado la experiencia, a través de un informe de evaluación formativa y dos estudios de caso sobre los "Espacios para Crecer" y el "Baúl de Juegos" (véase el apartado de bibliografía). En segundo lugar, la estrategia de coordinación de los facilitadores comunitarios se ha convertido en un elemento central del programa, mejorando el funcionamiento del mismo. Finalmente, se ha seguido la tarea iniciada por los facilitadores, diseñando actividades dirigidas al contexto comunitario y atendiendo a las necesidades especiales de las familias de excombatientes y las familias desplazadas por la violencia política en Colombia.

\section{Sostenibilidad y continuidad de la intervención}

Edúcame Primero, Colombia tiene dos componentes fundamentales: (a) las actividades psico-educativas para promover el desarrollo de competencias de los menores, mejorar el desempeño académico y prevenir el abandono de la escuela; y (b) los espacios de juego alternativos al trabajo infantil. Las dos estrategias centrales se han mantenido a lo largo de la experiencia, pese a los cambios que se han producido en el contexto de intervención. Las prácticas preventivas con menores en riesgo le han dado continuidad al programa en dos marcos de cooperación internacional diferentes, con Estados Unidos y con España, de forma sucesiva. También se ha producido un proceso de apropiación local del programa, que en el caso de Barranquilla pasó a estar coordinado por la Universidad del Norte con niveles adecuados de efectividad.

La participación de mediadores comunitarios resulta fundamental en la sostenibilidad del programa, aportando recursos naturales en el desarrollo de actividades preventivas. A la misma dinámica de sostenibilidad se ha incorporado posteriormente el diseño de un catálogo de acciones comunitarias y el sistema de micro-financiación de iniciativas locales. La continuidad del programa prevé la transferencia de actividades a Lima (Perú) y la participación de profesionales españoles de psicología en estancias breves de cooperación internacional.

\section{Lecciones aprendidas}

Esta experiencia ilustra cómo las estrategias de ajuste comunitario mejoran la implementación del programa y su sostenibilidad a medio plazo. La adecuación del programa a las comunidades en las que se desarrolla permite la continuidad de la intervención pese a los 
cambios institucionales, políticos, socio-culturales y demográficos del contexto de aplicación. Edúcame Primero, Colombia es un ejemplo de cómo la modificación de aspectos secundarios del programa (a) mejora la adaptabilidad a necesidades especiales, (b) potencia la efectividad al actuar sobre las dinámicas comunitarias y (c) garantiza la sostenibilidad de los componentes centrales de la intervención en diferentes contextos y con diferentes poblaciones.

La estrategia de los facilitadores comunitarios es posiblemente el mejor ejemplo de ajuste a los contextos de aplicación. No obstante, a partir de dicha experiencia, el programa ha ido incorporando acciones dirigidas a mejorar la cohesión social del vecindario, las dinámicas de implicación de la escuela y los procesos de socialización familiar. Entre las lecciones aprendidas, también hay que mencionar que el programa ha evolucionado desde las propuestas genéricas de "erradicación" del trabajo infantil a acciones específicas de carácter preventivo, centradas en las peores formas del trabajo infantil.

\section{Condiciones necesarias para la transferencia}

Los "Espacios para Crecer" y el "Baúl de Juegos" se apoyan en materiales educativos que facilitan su incorporación en los centros escolares. Las actividades están adaptadas a los diferentes niveles educativos, se han puesto a prueba de forma experimental y han incorporado cambios para ajustarse a las peculiaridades de cada país donde ha sido aplicado. Pese a que los materiales son efectivos y sensibles culturalmente, la calidad de la implementación depende en gran parte de la implicación del profesorado y las reacciones de la comunidad educativa en su conjunto. Para mejorar el impacto de la intervención se ha mostrado fundamental el trabajo coordinado con las familias, el intercambio de buenas prácticas entre aplicadores y las acciones de preparación comunitaria.

\section{Criterios de la OIT-IPEC para la prevención del trabajo infantil}

En 1992 se pone en marcha el Programa Internacional para la Erradicación del Trabajo Infantil (IPEC) de la Organización Internacional del Trabajo (OIT). EI IPEC ha establecido, en colaboración con los gobiernos, las líneas estratégicas de intervención en este ámbito y ha contribuido a reducir la prevalencia del trabajo infantil en el mundo en las últimas décadas. Para completar la descripción de la red de agentes comunitarios en la prevención del trabajo infantil en Barranquilla, a continuación revisamos de nuevo la experiencia utilizando el marco de referencia de la OIT. En primer lugar, describimos los componentes centrales de la intervención en el informe de buenas prácticas. En segundo lugar, analizamos las implicaciones de esta experiencia, en un apartado final de sistematización.

\section{Informe de buenas prácticas}

\section{¿En qué contexto se originó esta experiencia?}

Más de 14 millones de niños y niñas entre 5 y 17 años se dedican a algún tipo de actividad laboral en América Latina (OIT, 2010), de los cuales, más de 9 millones se dedican a trabajos 
considerados peligrosos o englobados en lo que se denominan Peores Formas de Trabajo Infantil. En América Latina se han desarrollado planes nacionales contra el trabajo infantil y se ha observado una reducción de la prevalencia del problema de en torno a un 1 por ciento entre 2004 y 2008 . La experiencia se dirigió a las zonas de actuación preferente en Barranquilla, en barrios tales como El Silencio, Los Olivos, Por Fin, Alboraya, Pumarejo, Palermo y Olaya Herrera. Estos barrios aglutinan gran parte de la población infantil dedicada a actividades laborales. Las formas de trabajo infantil más habituales consisten en colaborar en negocios familiares, la venta de productos en la calle y la mendicidad.

\section{¿Cómo surgió la iniciativa?}

El programa surge como una iniciativa de cooperación al desarrollo entre entidades norteamericanas y colombianas. Esta iniciativa forma parte de un conjunto de experiencias similares desarrolladas en América Latina en la última década, en respuesta a los planes nacionales e internacionales de lucha contra el trabajo infantil. "Edúcame Primero" responde a las prioridades del plan para la erradicación del trabajo infantil en Colombia y al Programa Internacional para la Erradicación del Trabajo Infantil (IPEC) de la Organización Internacional del Trabajo (OIT). En la iniciativa original participaron Partners of Americas, DevTech, MerciCorps y Cinde. El "Baúl de Juegos", por su parte, es una propuesta de Philippe Valls y Nicole Dagnino. La iniciativa del IPEC fue fundamental para la puesta en marcha de programas similares en la región. De ese modo, las prioridades políticas internacionales contribuyeron a la concienciación local sobre las necesidades y problemas de los niños y niñas trabajadores.

\section{¿Cuál fue el objetivo de la experiencia?}

El programa pretendía reducir la prevalencia de las peores formas de trabajo infantil en Barranquilla. Para ello ponía en marcha actividades educativas y de desarrollo personal de los niños y niñas, que pretendían mejorar el desempeño académico, prevenir el absentismo y el abandono escolar y fortalecer las competencias sociales y los recursos psicológicos de los participantes.

\section{¿Quién o quiénes se encargaron de la puesta en marcha?}

Desde 2010 la Universidad del Norte gestiona el programa con recursos propios. La iniciativa cuenta con un coordinador, que es miembro del CIDHUM, y una red de "facilitadores", que son estudiantes de psicología que coordinan la implementación del programa en cada centro educativo. La participación de facilitadores tiene una vertiente de fortalecimiento institucional, puesto que articula la relación de la Universidad del Norte con su entorno comunitario. Los estudiantes desarrollan competencias en el contexto de la atención de necesidades de la comunidad. Por su parte, la monitorización y la evaluación del programa son realizadas por un equipo internacional de investigadores del CIDHUM (Colombia) y del LRPC (España). El coordinador del LRPC supervisa al grupo de cooperantes españolas que realizan estancias internacionales en el proyecto. Las cooperantes españolas se integraron con el grupo de facilitadores colombianos, tanto en la implementación de actividades como en la evaluación del programa. 
¿Qué se hizo?

El programa consistió en la aplicación de los "Espacios para Crecer" y el "Baúl de Juegos" en 20 centros educativos de Barranquilla. Un grupo de 19 facilitadores trabajaron en red para coordinar la aplicación de actividades. Los mediadores comunitarios adaptaron el programa a cada centro educativo e implicaron a las familias en el desarrollo de las actividades. También se coordinaron entre sí para detectar buenas prácticas de intervención. Aquellas experiencias que funcionaron bien fueron trasladadas a otros contextos educativos. Los facilitadores se apoyaron mutuamente a lo largo del programa, resolviendo problemas de aplicación, aportando consejos y conformando un grupo de actores clave en el mantenimiento de la intervención.

\section{¿Cómo se hizo?}

El programa de cooperación internacional que sirvió de antecedente generó una red de centros colaboradores con la Universidad del Norte. El coordinador del programa en Colombia contactó con los centros educativos para mantener la intervención con la participación de estudiantes universitarios. Una vez formado el consorcio, los facilitadores lideraron la aplicación local del programa, monitorizando las actividades de los "Espacios para Crecer" y el "Baúl de Juegos". El contacto entre los facilitadores y los directivos de cada centro educativo resultó esencial en el funcionamiento del programa. Para el reclutamiento de los facilitadores se realizó una convocatoria abierta para profesionales de la educación y las ciencias sociales. Aquellos que fueron seleccionados recibieron por una formación intensiva en la estrategia pedagógica del Quantum Learning, así como en estrategias de seguimiento y evaluación de programas. Para seleccionar a los facilitadores se tuvo en cuenta que fueran personas capacitadas para la docencia y la formación con niños y jóvenes, y que fueran conocedores de los entornos comunitarios en los que se iban a implementar los espacios del programa.

\section{¿Para quién se hizo?}

El programa se aplicó a niños y niñas trabajadores de 5 a 16 años, residentes en los barrios de El Silencio, Los Olivos, Por Fin, Alboraya, Pumarejo, Palermo y Olaya Herrera. De forma genérica y gracias a la dinamización de los facilitadores, el impacto de la intervención alcanzó a las familias de los niños trabajadores, que estuvieron expuestas a un proceso de transformación y aprendizaje. Uno de los resultados más significativos de la acción de los facilitadores fue la dinamización de la relación entre las familias y los centros educativos. En algunos colegios se observó un proceso de fortalecimiento institucional. Fueron los casos en los que la comunidad educativa se apropió del programa de prevención, e implicó al profesorado en los objetivos de lucha contra el trabajo infantil. De hecho, esta estrategia sirvió para definir estrategias formales de implicación de la red de colegios en el desarrollo del programa.

\section{¿Con quién se hizo?}

La Universidad del Norte colaboró con 20 centros educativos. Un total de 19 estudiantes de la Universidad del Norte participaron como facilitadores. La Universidad de Sevilla aportó 8 cooperantes internacionales. También participó en la experiencia un becario postdoctoral de la Agencia Española de Cooperación Internacional (AECID), que coordinó las actividades de evaluación bajo la supervisión 
del equipo internacional de trabajo.

\section{¿Cuánto costó?}

En su primera aplicación, "Edúcame Primero, Colombia" contó con un presupuesto de cinco millones de dólares para el desarrollo del programa a lo largo de tres años en toda Colombia, con el objetivo de recibir unos 10.200 niños y jóvenes. Sin embargo, tras la apropiación de la iniciativa por la entidad local, el programa carece de un presupuesto específico. La iniciativa que describimos en este documento se basó en la participación de los estudiantes, los recursos organizativos de la universidad y los recursos informales de la comunidad. La colaboración de la Universidad de Sevilla ascendió a unos 10.000 euros en el período analizado.

\section{¿Cómo se hizo el seguimiento, monitoreo y evaluación?}

Se realizó una evaluación de resultados y una evaluación formativa. Los facilitadores realizaron una monitorización continua del programa. La evaluación de resultados fue realizada por la Universidad del Norte. La evaluación de resultados consistió en una encuesta de seguimiento a las familias participantes, en la que se valoró el rendimiento académico, la asistencia a la escuela y las prácticas de trabajo infantil de los niños participantes en el programa. Además, se aplicaron escalas de socialización, habilidades sociales y habilidades cognitivas a los niños y niñas participantes en el programa, antes de su ingreso y una vez finalizada la intervención, para observar los cambios y el impacto del programa en estos aspectos. La evaluación formativa fue realizada por un equipo internacional de la Universidad de Sevilla y la Universidad del Norte. La evaluación formativa consistió en un análisis detallado del proceso de aplicación del programa. En el marco de dicha evaluación se introdujeron cambios en el programa y se potenció el carácter comunitario de las acciones. Los facilitadores se ocuparon de supervisar el proceso de aplicación del programa, tomando decisiones periódicamente sobre el funcionamiento de la intervención e introduciendo de forma continua ajustes en el programa.

\section{¿Cuáles fueron los resultados logrados?}

El programa aumentó el tiempo de dedicación de los niños y niñas a actividades académicas, de juego y de socialización entre iguales. La intervención se mostró efectiva en la mejora del rendimiento académico, y en la reducción del absentismo y el abandono escolar. También se observaron resultados positivos en el desarrollo de competencias personales de los participantes, como mostraron los indicadores referidos a las habilidades sociales y la autoestima.

\section{¿Cuáles fueron las limitaciones?}

La intervención se basa en la aplicación de actividades educativas previamente diseñadas. El proceso de implementación depende en la práctica de la reacción del sistema educativo. Por ejemplo, en ocasiones los profesores cuestionaron la pertinencia del programa o simplemente tenían prioridades diferentes sobre las necesidades de los niños. Como los materiales educativos no formaban parte del plan de centro, en algunos casos surgieron barreras en la aplicación del programa. En definitiva, se hacía necesario un enfoque comunitario de intervención, que tuviera en cuenta la apropiación de las estrategias de prevención del trabajo infantil por los centros escolares. 
¿Qué oportunidades se presentaron?

La experiencia de los facilitadores reveló que el trabajo con las familias resultaba fundamental para el buen desarrollo del programa. A lo largo de la implementación se observó que la relación de las familias con la escuela es un proceso clave, que debería incorporarse entre las acciones estratégicas de forma planificada. En otras palabras, las familias, las escuelas y, de forma amplia, la comunidad ofrecen oportunidades para potenciar la efectividad de las actividades preventivas. Los facilitadores realizaron informalmente dichas actividades en el marco del programa. Además, intercambiaron entre sí las experiencias que habían funcionado adecuadamente.

¿Qué recomendaría a otras instituciones que quieren poner en marcha una experiencia similar?

En primer lugar, se recomienda realizar una aplicación flexible de las actividades educativas, adaptando los contenidos del programa a las peculiaridades de cada contexto. En segundo lugar, esta experiencia ilustra el valor de un enfoque comunitario de intervención, que incide en los contextos en los que se desarrolla la vida de los niños y niñas participantes en el programa. Finalmente, resulta práctico contar con un espacio de encuentro entre los aplicadores del programa, donde puedan intercambiar recursos y experiencias a lo largo de la intervención. De esa forma se refuerza la integridad de la acción y se incorpora un componente formativo a la intervención.

\section{¿Qué condiciones permitirían replicar esta experiencia en otro lugar?}

Los materiales educativos han sido comprobados previamente y están diseñados para los diferentes niveles académicos. Además, han pasado por un proceso de adaptación cultural antes de aplicarlo en cada país. Para facilitar su aplicación, es recomendable que cada organización educativa lo incorpore al Plan de Centro, generando un consenso previo entre el profesorado. También se recomienda la participación de las asociaciones de madres y padres de alumnos/as, para facilitar el control comunitario de la intervención.

\section{Sistematización de experiencias exitosas}

¿Por qué esta experiencia puede ser de interés para otras organizaciones?

La participación de mediadores comunitarios en la iniciativa "Edúcame Primero, Colombia" es un ejemplo de cómo un programa puede modificarse de manera sustancial, y mantener al mismo tiempo la integridad de la intervención. El programa introduce adaptaciones en función del contexto $y$, en consecuencia, se aplica de un modo diferente en diferentes escuelas, en comunidades diversas y con distintas poblaciones. Sin embargo, las estrategias centrales se mantienen, garantizando la implementación del modelo de prevención del trabajo infantil que inspira el programa. Eso pone de manifiesto que en la aplicación del programa resulta más decisivo la sostenibilidad de las estrategias básicas de la intervención que replicar el plan de acción con exactitud o utilizar los materiales educativos respetando fielmente el original.

La experiencia puede ser de interés para otras organizaciones por poner el acento en la innovación local (a partir de los elementos básicos del programa), por encima de la replicación de una serie de actividades muy definidas a priori. La experiencia de los mediadores comunitarios también proporciona algunos ejemplos de cómo realizar el ajuste comunitario con los centros escolares, los 
barrios y las familias participantes.

¿Qué evidencias existen sobre los beneficios de la experiencia?

La evaluación de resultados de "Edúcame Primero, Colombia" durante su aplicación 20082010 demostró que el programa mejora el rendimiento académico, reduce el absentismo escolar, aumenta la autoestima y las habilidades sociales, disminuye el número de asignaturas no superadas y afecta negativamente al número de horas de trabajo infantil. Los "Espacios para Crecer" se aplicaron además como una práctica basada en la evidencia, de la que existía registro previo de su efectividad. La intervención mostró un impacto positivo muy claro en la mejora del rendimiento en matemáticas y lenguaje.

Durante el curso 2010-2011 se realiza una evaluación formativa que demuestra el impacto positivo de los facilitadores comunitarios en la mejora de la implementación del programa y en la obtención de resultados. Los facilitadores resultan claves para conseguir la implicación de las familias y para incorporar el programa en la dinámica de los centros escolares.

Recientemente, una investigación con datos del programa ha demostrado la relación entre trabajo infantil y rendimiento académico. Las condiciones laborales, el número de horas y la jornada matinal son los factores que más negativamente afectan al desarrollo académico de los niños que trabajan. Véase el apartado de referencias para consultar las evidencias del programa. Todos los documentos están disponibles a solicitud de los interesados.

¿Se ha puesto en marcha esta experiencia en otra zona o contexto? ¿Cuáles han sido los resultados obtenidos?

Primero, entre 2004 y 2008 el programa Primero Aprendo fue aplicado en 6 países de CentroAmérica: Costa Rica, República Dominicana, El Salvador, Guatemala, Honduras y Nicaragua. La evaluación de las intervenciones permitió identificar 14 prácticas educativas efectivas contra el trabajo infantil. Entre ellas se contaban los "Espacios para Crecer".

En segundo lugar, se realizó una implantación piloto de los "Espacios para Crecer" en República Dominicana. La evaluación permitió comprobar la efectividad de la intervención. Entre 2008 y 2010 se aplicó el programa "Edúcame Primero, Colombia" (que incluye los "Espacios para Crecer"), en diferentes regiones del país, con resultados positivos. Desde 2010 la Universidad del Norte y la Universidad de Sevilla han mantenido el "Baúl de Juegos", junto con otras actividades psico-educativas, en Barranquilla. En todos los casos el programa se ha mostrado positivo para reducir el abandono escolar, con repercusiones en las peores formas de trabajo infantil.

\section{¿Continuarán los beneficios en un largo plazo?}

Precisamente una de las características llamativas de la aplicación en el caribe colombiano ha consistido en la continuidad del programa en diferentes contextos institucionales. En este caso la sostenibilidad de la intervención se relaciona con (a) la apropiación local del programa, (b) el mantenimiento de los componentes centrales con diferentes consorcios y agentes de cooperación internacional y (c) la utilización de recursos naturales de la comunidad, con la participación de facilitadores comunitarios. Por su parte, es de esperar que el nuevo enfoque en el desarrollo de competencias de las escuelas y las comunidades implicadas contribuyan a que se mantengan los 
beneficios a largo plazo.

¿Coincide la experiencia con las prioridades nacionales en este ámbito?

El programa sigue las recomendaciones y prioridades del Programa Internacional para la Erradicación del Trabajo Infantil (IPEC) de la Organización Internacional del Trabajo (OIT), junto con las guías del Comité Interinstitucional para la Erradicación del Trabajo Infantil y la Protección del Trabajo Juvenil (CIETI) en Colombia. De acuerdo con los sucesivos planes de políticas públicas en Colombia, desde mediados de la década de los 1990 hasta la actualidad, esta intervención ha contribuido a (a) visibilizar el problema del trabajo infantil en Colombia, sensibilizando a la población, las organizaciones privadas y las instituciones públicas; y (b) prevenir y eliminar las peores formas del trabajo infantil, desarrollando una política de carácter nacional y descentralizada. Véase, por ejemplo, el documento de la OIT "Desarrollo de la política pública para la prevención y erradicación del trabajo infantil en Colombia" (2009).

¿Hubo una consulta con diferentes actores antes de la puesta en marcha de la experiencia?

El consorcio formado por Partners of the Americas, Devtech, Cinde y Mercy corps fue el promotor inicial del programa en Colombia. A continuación contactaron con organizaciones no gubernamentales colombianas para implementar el programa en todo el ámbito nacional. En los tres años de aplicación del programa se llevaron a cabo 370 "Espacios para Crecer", alcanzando a un total de 9.582 niños y niñas. Entre las entidades participantes cabe mencionar ACJ Bogotá, CACBosa, Fundación Renacer, Corpoeducación, Fundación "Volver a la Gente”, Fundación Mamonal, Fundación Universidad del Norte, Fundación para el desarrollo del niño, la familia y la comunidad, Fundación El camino, Cedecur y Fe y Alegría.

Se realizaron contactos previos con los centros educativos antes de aplicar el programa. También se contactó con el Instituto de Bienestar Familiar. Los contactos iniciales sirvieron para explicar los contenidos del programa y recabar el apoyo de los centros. Para ello se explicó la importancia de prevenir el trabajo infantil desde el contexto educativo. En ocasiones se realizaron sesiones de formación del profesorado antes de aplicar el programa. Posteriormente se explicaba a marcha del programa y los resultados iniciales, convocando para ello a los padres y madres del alumnado junto con el profesorado. Con la incorporación de los facilitadores, el contacto con las escuelas y las familias fue continuado.

¿Cómo se hizo para optimizar los recursos humanos, financieros y materiales?

El programa se inició como un proyecto de cooperación internacional, con ayuda para el desarrollo y la participación de organizaciones colombianas y norteamericanas. Después de esa primera experiencia de aplicación, hay dos aspectos que optimizaron la gestión de los recursos disponibles. Primero, la apropiación local por parte de la Universidad del Norte hace que el programa se integre en la dinámica de la universidad, contando con recursos propios. Aunque no se defina formalmente como tal, "Edúcame Primero, Colombia" funciona como una iniciativa de aprendizajeservicio, en la que los estudiantes combinan el desarrollo de competencias profesionales con el servicio a la comunidad. Los recursos informales de los estudiantes se ponen a disposición de la población. Segundo, la estrategia de facilitadores comunitarios también se basa en recursos 
naturales, mejorando la eficiencia y la continuidad de la intervención.

\section{Referencias Bibliográficas}

-Bosque, R. (2013). "Baúl de Juegos": La semilla de una intervención social y comunitaria para la prevención y erradicación del trabajo infantil en Barranquilla. Trabajo Fin de Máster (TFM) del Máster en Psicología de la Intervención Social y Comunitaria de la Universidad de Sevilla.

-Chapa, E., D., Ramos, I. \& Maya-Jariego, I. (2012). "Baúl de Juegos": una iniciativa lúdica para reducir la incidencia y los efectos del trabajo infantil en menores de Barranquilla. Maya-Jariego, I. \& Palacio, J. E. (Eds). Edúcame Primero Colombia. Un espacio de colaboración entre la Universidad de Sevilla y la Universidad del Norte. Sevilla: Oficina de Cooperación al Desarrollo de la Universidad de Sevilla.

-Chapa de la Peña, M. E. (2012). Las redes personales y la familia: el caso de los niños inscritos en el programa Baúl de Juegos en Barranquilla (Colombia). Trabajo Fin de Máster (TFM) del Máster en Psicología de la Intervención Social y Comunitaria de la Universidad de Sevilla.

-Cortés, V. (2013). Gestión del cambio en organizaciones educativas. Estudio de caso de tres centros donde se aplica "Baúl de Juegos". Trabajo Fin de Máster (TFM) del Máster en Psicología de las Organizaciones y del Trabajo de la Universidad de Sevilla.

-Holgado, D., Maya-Jariego, I. Palacio, J., Ramos, I., Oviedo-Trespalacios, O. Romero-Mendoza, V. \& Amar, J. (2014). Impact of Child Labour in Academic Performance: Evidence from the Program "Edúcame Primero, Colombia". International Journal of Educational Development, 34, 58-66.

-Holgado, D., Maya-Jariego, I., Palacio, J. \& Oviedo-Trespalacios, O. (2014). Two profiles of child labor: relocated children to suburban areas compared to the key role of social and labor characteristics of mothers in urban settings. En revisión.

-Holgado, D., Maya-Jariego, I. \& Palacio, J. E. (2012). “Espacios para Crecer”: un caso de intervención comunitaria en trabajo infantil desde el contexto educativo. Maya-Jariego, I. \& Palacio, J. E. (Eds). Edúcame Primero Colombia. Un espacio de colaboración entre la Universidad de Sevilla y la Universidad del Norte. Sevilla: Oficina de Cooperación al Desarrollo de la Universidad de Sevilla.

-Holgado, D., Maya-Jariego, I., Ramos, I. \& Palacio, J. (2014). El papel de los facilitadores en la implementación de los "Espacios para Crecer": evaluación formativa del programa con menores trabajadores "Edúcame Primero, Colombia". Universitas Psychologica, 13 (4).

-Márquez López, E. (2011). Estrategias de implicación comunitaria en programas de cooperación al desarrollo para la erradicación del trabajo infantil en Sudamérica. Evaluación de la experiencia de los Espacios para Crecer en Barranquilla (Colombia). Trabajo Fin de Máster (TFM) del Máster en Psicología de la Intervención Social y Comunitaria de la Universidad de Sevilla.

-Maya-Jariego, I. \& Palacio, J. E. (Eds) (2012). Edúcame Primero Colombia. Un espacio de colaboración entre la Universidad de Sevilla y la Universidad del Norte. Sevilla: Oficina de Cooperación al Desarrollo de la Universidad de Sevilla. 